\title{
Erratum
}

\section{Erratum. National Institutes of Health-Sponsored Clinical Islet Transplantation Consortium Phase 3 Trial: Manufacture of a Complex Cellular Product at Eight Processing Facilities. Diabetes 2016;65:3418-3428}

Camillo Ricordi, Julia S. Goldstein, A.N. Balamurugan, Gregory L. Szot, Tatsuya Kin, Chengyang Liu, Christine W. Czarniecki, Barbara Barbaro, Nancy D. Bridges, Jose Cano, William R. Clarke, Thomas L. Eggerman, Lawrence G. Hunsicker, Dixon B. Kaufman, Aisha Khan, David-Erick Lafontant, Elina Linetsky, Xunrong Luo, James F. Markmann, Ali Naji, Olle Korsgren, Jose Oberholzer, Nicole A. Turgeon, Daniel Brandhorst, Andrew S. Friberg, Ji Lei, Ling-jia Wang, Joshua J. Wilhelm, Jamie Willits, Xiaomin Zhang, Bernhard J. Hering, Andrew M. Posselt, Peter G. Stock, and A.M. James Shapiro

https://doi.org/10.2337/db17-er09a

In the article listed above, Xiaojuan Chen, now of the Columbia Center for Translational Immunology, Columbia University, New York, NY, was erroneously omitted from the author list.

Dr. Chen contributed to the Clinical Islet Transplantation (CIT) trial as director of the islet processing team at the Comprehensive Transplant Center, Northwestern University Feinberg School of Medicine (Chicago, IL). Her direct involvement resulted in 15 patients receiving islet transplants at Northwestern University. She specifically supervised the technical staff of the islet team on every aspect of islet processing procedure, which led to the successful utilization of the SERVA enzymes for pancreas digestion and islet recovery. She also participated in the development of the modified glucose-stimulated insulin release assay used by the CIT trial for islet quality evaluation in vitro.

The authors apologize for this unfortunate omission. The online version has been updated to correct this omission. 